\title{
Unfair Noisy Channels and Oblivious Transfer
}

\author{
Ivan Damgård ${ }^{1}$, Serge Fehr ${ }^{2 \star}$, Kirill Morozov ${ }^{1}$, and Louis Salvail ${ }^{1 \star \star}$ \\ $1 \mathrm{BRICS}^{\star \star \star}, \mathrm{FICS}^{\dagger}$, Aarhus University, Denmark \\ \{ivan,kirill, salvail\}@brics.dk \\ $2 \mathrm{ACAC}^{\ddagger}$, Department of Computing, Macquarie University, Australia \\ sfehr@ics.mq.edu.au
}

\begin{abstract}
In a paper from EuroCrypt'99, Damgård, Kilian and Salvail show various positive and negative results on constructing Bit Commitment (BC) and Oblivious Transfer (OT) from Unfair Noisy Channels (UNC), i.e., binary symmetric channels where the error rate is only known to be in a certain interval $[\gamma . . \delta]$ and can be chosen adversarily. They also introduce a related primitive called PassiveUNC. We prove in this paper that any OT protocol that can be constructed based on a PassiveUNC and is secure against a passive adversary can be transformed using a generic "compiler" into an OT protocol based on a $U N C$ which is secure against an active adversary. Apart from making positive results easier to prove in general, this also allows correcting a problem in the EuroCrypt'99 paper: There, a positive result was claimed on constructing from UNC an OT that is secure against active cheating. We point out that the proof sketch given for this was incomplete, and we show that a correct proof of a much stronger result follows from our general compilation result and a new technique for transforming between weaker versions of OT with different parameters.
\end{abstract}

\section{Introduction}

Bit Commitment (BC) and Oblivious Transfer (OT) are the most fundamental primitives in cryptographic protocol design 81/3|9 10. But in a scenario with only two players, neither primitive can be implemented with unconditional security based only on standard, error free communication. Even quantum communication does not help [14 13. However, Crépeau and Kilian have shown that both primitives can be implemented based on a binary symmetric channel (BSC) 55. A BSC is a channel for transmitting single bits, and for every bit transmitted, the channel decides with some fixed probability to flip the bit before it is

* Most of this work was done while at BRICS, Aarhus University, Denmark.

$\star \star$ Research funded by European project PROSECCO.

$\star \star \star$ Basic Research in Computer Science (www.brics.dk), funded by the Danish National Research Foundation.

$\dagger$ FICS, Foundations in Cryptography and Security, funded by the Danish Natural Sciences Research Council.

$\ddagger$ Centre for Advanced Computing - Algorithms and Cryptography. 
given to the receiver. Unfortunately, results based on BSCs do not give realistic security guarantees. The reason for this is that one must expect that a cheating player will try to influence the channel and have this work to his/her advantage, for instance by lowering the noise rate in order to learn more than expected about what the other party sent or received. Note that one can always hide the fact that the channel was made less noisy by pretending to have sent(received) a more noisy signal than the one actually sent(received). Moreover, even in the absence of such attacks, it is hardly realistic to assume that the noise rate is known exactly.

In [7, Damgård, Kilian and Salvail introduce the Unfair Noisy Channel (UNC) as a model of a noisy channel that is more realistic in cryptographic applications than a BSC. A $(\gamma, \delta)$-UNC is basically a BSC, where, however, the noise rate is only known to be in a certain interval $[\gamma . . \delta]$, and where if the sender or receiver has been corrupted by an adversary, the adversary can set the noise rate to any desired value in the interval. So a UNC models active cheating directed against the way a physical channel works in order to manipulate the error rate. If the channel is a radio link, for instance, the adversary could invest in more sophisticated receiving equipment without telling the other party and thereby lowering the noise rate from his point of view. However, it may still be realistic to assume that he cannot remove all noise from the channel, so such a case can be captured in the UNC model.

Another primitive was also introduced, namely a $(\gamma, \delta)$-PassiveUNC. This is a BSC with error rate $\delta$, but where the adversary gets for every transmission some side information $z$ with the property that given $z$, the bit received/sent by the other (honest) player can be guessed with error probability $\gamma$. In other words, knowledge of $z$ brings the error rate down to $\gamma$ from the adversary's point of view. This models a passive, i.e., "honest but curious" adversary, who measures somewhere "in the middle" of the channel, and then later uses the information obtained to compute data he should not have access to.

In [7, it was proved that Bit Commitment (BC) can be implemented with unconditional security based on a $(\gamma, \delta)$-UNC if and only if the interval $[\gamma . . \delta]$ is not too wide, more precisely, if and only if $\delta<2 \gamma(1-\gamma)$. It was also shown that one cannot base Oblivious Transfer (OT) on a $(\gamma, \delta)$-UNC (nor on a PassiveUNC) if $\delta \geq 2 \gamma(1-\gamma)$. On the positive side, it was shown that if $\gamma$ and $\delta$ satisfy a rather complex condition (stronger than $\delta<2 \gamma(1-\gamma)$ ), then OT (with passive security) can be based on a $(\gamma, \delta)$-PassiveUNC.

Finally, it was claimed that this same result also holds when using a $(\gamma, \delta)$ UNC, and with security against active cheating. This was based on a standard idea where the players use bit commitments to commit to all private data, including what is sent and received on the channel, and then use generic zero-knowledge techniques to demonstrate that they follow the protocol. This technique indeed works assuming that we can force a cheating player to commit to the bits he actually sends or receives over the channel (except with arbitrarily small probability). This assumption is true for a BSC: for instance the sender $S$ can be instructed to commit to bits $b_{i}, i=1 . . n$, and send them over the BSC with 
noise rate, say, $\delta$. Having received bits $\hat{b}_{i}, i=1 . . n$, the receiver $R$ then asks to have all committed bits opened except one, say $b_{j}$. If $S$ was honest, we expect that a fraction of about $\delta$ of the opened bits will be different from the received bits $\hat{b}_{i}, i=1$..n. So $R$ is instructed to reject if the fraction of disagreement is significantly larger than $\delta$. If $R$ does not reject, this means intuitively that he believes that the committed bit $b_{j}$ really is the bit that was sent over the channel and resulted in $R$ receiving $\hat{b}_{j}$. This is justified since it follows from standard probability theory that the probability of having $b_{j}$ different from the $j$ 'th bit actually sent and still have the receiver accept, can be made arbitrarily small by increasing $n$.

Unfortunately, no such technique can work for a UNC. We show below that for any protocol that aims to implement a "committed UNC", the probability of error is at least a constant, namely $(\delta-\gamma) /(1-2 \gamma)$. This problem was not taken care of in [7].

In this paper, we show a different (and correct) way to apply the idea of using commitments and zero-knowledge proofs to enforce correct behavior. This turns out to lead to a result that is much more general than what was claimed in 7] and which can be informally stated as follows: Any two-party protocol that, based on a $(\gamma, \delta)$-PassiveUNC, implements an OT secure against passive cheating, can be transformed using a generic "compiler" into a protocol that uses a $(\gamma, \delta)$-UNC for communication and builds an OT secure against active cheating.

The opposite direction of this result is also true, and trivial to prove. So this implies that, to prove positive or negative results, on building OT from UNC or PassiveUNC, we can now concentrate only on the case of PassiveUNC and passive cheating - which is clearly much simpler. It also immediately implies a complete proof of the claim made in [7].

In the final part of the paper we exploit this, and a new technique for transforming between the weaker versions of OT, in order to prove a stronger positive OT result than the one claimed in [7. In other words, there is now a much larger range of $(\gamma, \delta)$-values for which we can implement OT based on a $(\gamma, \delta)$-UNC. For instance we can now show that robust OT follows from a $(\gamma, \delta)$-UNC with any value of $\delta$ between 0 and $1 / 2$, provided $\gamma$ is close enough to $\delta$.

Due to space limitations, some proofs could not be included in this proceedings version of the paper. They can be found in the full paper [6].

\section{Models of Communication and Adversaries}

Our protocols throughout the paper take place in a model with two players $A, B$ connected by an error free channel and also by a noisy channel with some particular characteristic, such as a UNC or a PassiveUNC. We assume a bounded delay in message delivery for all channels such that failure to send a message can be detected.

In order to specify formally the channels and reductions we study, we will use the universally composable framework of Canetti [2]. In this framework, players 
in a protocol can be given access to one or more ideal functionalities. Such a functionality can be thought of as a trusted party $T$ with whom every player can communicate privately. There is a number of commands specified that $T$ will execute. Every player can send a command to $T$, and $T$ will faithfully carry out the command according to its specification, and may send results back to (some of) the players. Many cryptographic constructions - including ours - actually aim at building a protocol for the players only (without a trusted party) that does "the same thing" as some ideal functionality $T$, even if an adversary can corrupt some of the players and make them behave as he likes. The framework provides a precise definition of what it means that a protocol $\pi$ in this way securely implements $T$. If this definition is satisfied, then any protocol that is secure when using $T$ is also secure if $T$ is replaced by $\pi$. In its full generality, the definition is robust against adaptive adversaries and concurrent composition of protocols.

All our protocols are in the 2-player case with information theoretic security. Here, the standard approach in previous research to security proofs has been to assume that either $A$ or $B$ is cheating, then prove some relevant security properties, and finally to prove that if both parties are honest, then the protocol "works correctly". We express this in the UC framework by assuming an infinitely powerful non-adaptive adversary who from the start has corrupted no one, or either $A$ or $B$. While we believe that our results extend to adaptive adversaries, we do not prove or claim this in this paper. Furthermore, if the noisy channel is a UNC, then the adversary is assumed to be active, i.e., can decide the corrupted player's behavior. If the channel is a PassiveUNC, the adversary is passive.

Another consequence of being in the two-player case, is that we do not think of our protocols as subroutines in a multiplayer protocol, nor are we worried about external observers, only about what a corrupted $A$ or $B$ might do or learn. We therefore assume that unless the adversary corrupts a player, he gets no information about the communication between $A$ and $B$. At the cost of more complex proofs, our results extend to the case where the adversary always eavesdrops the error free channel.

To prove that a protocol $\pi$ satisfies the UC definition, one has to construct, for every adversary $A d v$ attacking the protocol in question, an ideal model adversary, or simulator $S$, which gets to attack an ideal scenario where only the players and $T$ are present. The goal of $S$ is to achieve "the same" as Adv could have achieved by an attack on the real protocol. In the framework, this is formalized by assuming an environment machine $Z$ which can communicate in a real life attack with $A d v$ and the honest players, and in the ideal model with $S$ and the honest players. The protocol is said to be secure if for every adversary $A d v$ there exists a simulator $S$, such that $Z$ cannot tell if it is in the real-life or the ideal model. For details, see [2].

In proofs of this type of security, $S$ usually works by running internally a copy of the adversary $A d v$, and passing interaction back and forth between $Z$ and $A d v$ with no change. If $S$ can simulate with an indistinguishable distribution both the view of $A d v$ attacking $\pi$ and simultaneously make the input/output 
behavior of the honest players be as in the real attack, then $Z$ will not be able to tell any difference.

The noisy channels we study in this paper can very conveniently be modeled as ideal functionalities, and reductions that build one type of channel from another can be proved secure in this framework. Since the results we prove are information theoretic in nature, we modify the UC model as given in [2] by allowing our adversaries and simulators infinite computing power - but we stress that honest players can execute our protocols efficiently.

\section{Some Functionalities}

We can now specify our basic types of channels precisely but for completeness we start by describing the functionality for standard (1-out-of-2) OT as well as for a weak version as introduced in [7] with parameters $0 \leq p, q \leq 1$ and $0 \leq \epsilon \leq 1 / 2$ :

\section{Functionality OT}

Send $\left(b_{0}, b_{1}\right)$ : The issuer of the Send command is called the sender, the other party is the receiver. On receipt of this command, the functionality records $\left(b_{0}, b_{1}\right)$ and outputs "which bit?" to the receiver. It ignores all further commands until the receiver sends a "Choice" command.

Choice $c$ : Receiving this command from the receiver, the functionality sends $b_{c}$ to the receiver if $c \in\{0,1\}$ and otherwise ignores the command.

For later convenience, we call the receiver's choice $c$ the selection bit and the bit $b_{1-c}$ (which is not revealed to the receiver) the secret bit.

Functionality $(p, q, \epsilon)$-WOT

Send $\left(b_{0}, b_{1}\right)$ : The functionality's action on this command is the same as in OT.

Choice $c$ : If $c \notin\{0,1\}$ then the functionality ignores the command. Otherwise, it chooses $\tilde{b}_{c} \in\{0,1\}$ such that $\operatorname{Pr}\left(\tilde{b}_{c} \neq b_{c}\right)=\epsilon$ and sends it to the receiver. Additionally, if the sender is corrupted, then with probability $p$ it sends $c$ to the sender, and if the receiver is corrupted, then with probability $q$ it sends $b_{1-c}$ to the receiver.

A $(\gamma, \delta)$-UNC is specified by the following functionality.

\section{Functionality $(\gamma, \delta)$-UNC}

Send $b$ : The issuer of the Send command is called the sender, the other party is the receiver. On receipt of this command, the functionality records $b$ and outputs a string "which error probability?" to the adversary. It ignores all further commands until the adversary sends an "Error probability" command.

Error probability $\epsilon$ : Receiving this command from the adversary, the functionality checks if $\gamma \leq \epsilon \leq \delta$. If not, the command is ignored. Otherwise, it chooses a random bit $b^{\prime}$, such that $\operatorname{Pr}\left(b^{\prime}=1\right)=\epsilon$, and sends $\hat{b}=b \oplus b^{\prime}$ to the receiver. 
What we want to model here is intuitively that a corrupted player may influence the error rate or even block the channel. But if both players are honest, transmissions will always go through, however, the error rate will fluctuate in some arbitrary way in the given interval. We therefore assume throughout about the adversary that if both players are honest, then the adversary will always give a legal error probability back when receiving a request from the UNC.

As mentioned, the adversary is allowed to set the error probability to any value in $[\gamma . \delta]$ for every transmission. However, if the adversary corrupts a player, any attack he can do following, say, algorithm $A l g$ can be simulated perfectly by an adversary that sets the error rate to $\gamma$ always, but adds artificial noise to any bit sent(received) in case $A l g$ wanted a larger error rate. We may therefore always assume that an active adversary who corrupts $A$ or $B$ always sets the error rate of the UNC to $\gamma$.

We introduce some notation that will be convenient: if we cascade a BSC with error rate $x$ and a BSC with error rate $y$, the result is again a BSC, we define $x \boxplus y$ to be the resulting error rate, $x(1-y)+(1-x) y$. Note that the operator $\boxplus$ is commutative, associative and satisfies that if $\left|x-x^{\prime}\right|<\nu$, then $\left|x \boxplus y-x^{\prime} \boxplus y\right|<\nu$ for all $y$.

\section{Functionality $(\gamma, \delta)$-PassiveUNC}

Send $b$ : The issuer of the Send command is called the sender, the other party is the receiver. On receipt of this command, the functionality chooses random bits $b^{\prime}, b^{\prime \prime}$, such that $\operatorname{Pr}\left(b^{\prime}=1\right)=\gamma$ and $\operatorname{Pr}\left(b^{\prime \prime}=1\right)=\nu$, where $\nu \boxplus \gamma=\delta$. This ensures that $\operatorname{Pr}\left(b^{\prime} \oplus b^{\prime \prime}=1\right)=\delta$. The functionality sends $\hat{b}=b \oplus b^{\prime} \oplus b^{\prime \prime}$ to the receiver. If the adversary has corrupted a player, it sends to the adversary a bit $z$, where $z=b \oplus b^{\prime \prime}$ if the sender is corrupted, and $z=b \oplus b^{\prime}$ if the receiver is corrupted. Intuitively, given $z$, the noise rate goes down to $\gamma$.

We need to consider the use of commitments and zero-knowledge proofs in our protocols. This can also be modeled by an ideal functionality, where one commits simply by giving the bit to the trusted party, who will then later open it on request from the committer. Furthermore, the trusted party will confirm that committed bits satisfy a given formula, if this is indeed true.

\section{Functionality Commit-and-prove ( $\mathrm{CaP})$}

Commit $c I D, b$ : Receiving this command, where $c I D$ is a bitstring and $b$ is a bit, do as follows: if no message containing $c I D$ has been received yet, record the value of $c I D, b$ and send as output Commit, $c I D$ to all players.

Open $c I D$ : if $c I D, b$ has been received earlier from the player issuing this command, send $b$ to all players.

Prove $L, \Phi$ : Receiving this command, where $L$ is a list of bit strings and $\Phi$ is a Boolean formula, check if $L$ contains only strings that has been used as identifiers for bits committed to by the issuer of the Prove command. If so, 
find the corresponding bits and check if they satisfy $\Phi$. If so, sends $(O K, L, \Phi)$ to all players. Else, send (Fail, $L, \Phi)$.

As bit commitment scheme in our protocols, we will use the UNC-based construction from [7], which works assuming $\delta<2 \gamma(1-\gamma)$ which we will assume throughout. This scheme is statistically close to perfect, regardless of $A$ and $B$ 's computing power. Furthermore, given any commitment scheme, one can always construct a new one, where one can prove in zero-knowledge that committed bits satisfy a given Boolean formula (see [1]). It follows that in any protocol where we assume access to a UNC, we may assume also a CaP without loss of generality.

A final functionality that will come in handy is the ability to choose random bits and numbers with a prescribed distribution:

\section{Functionality RandomChoice}

Flip $s I D, \nu$ : Here $s I D$ is a session ID and $\nu$ must be a probability. Once the functionality has received this command from every player containing identical values of $s I D, \nu$, it chooses a bit $b$ at random such that $\operatorname{Pr}(b=1)=\nu$ and sends $b$ to all players.

Uniform, $s I D, j$ : Here $s I D$ is a session ID and $j$ must be a natural number. Once the functionality has received this command from every player containing identical values of $s I D, j$, it chooses $i$ uniformly from $[0 . . j-1]$ and sends $i$ to all players.

Using standard techniques, one can implement this functionality based on the $\mathrm{CaP}$, with a statistically good simulation. It should be noted that in our twoplayer scenario, functionalities such as RandomChoice can only be realized if the adversary is allowed to abort after seeing the output. But this is consistent with the UC framework, where adversary and simulator are indeed allowed to abort any time.

\section{Committed (Passive)UNC}

We first define informally the notion of a committed $U N C$. This is a protocol for players $A, B$, using a $(\gamma, \delta)$-UNC and an error free channel. We will assume that $\delta<2 \gamma(1-\gamma)$, so that bit commitment can be done, based on the UNC. Note that if the UNC can only send bits from $A$ to $B$, we can still simulate a UNC in the opposite direction using the error free channel, so that we can assume that both $A$ and $B$ can commit to bits without loss of generality.

Intuitively, the purpose of a committed UNC is to act just like an ordinary UNC, but such that players are committed to the bits they send/receive on the UNC, at least except with some bounded probability.

We now define this concept more formally: a committed UNC protocol may halt because $A$ or $B$ reject. Otherwise it outputs two commitments, one from $A$ containing a bit $b_{A}$, and one from $B$ containing a bit $b_{B}$. Finally, the output designates one of the transmissions that were made over the UNC from $A$ to $B$. Let $s_{A}$ resp. $r_{B}$ be the bit sent, respectively received in this transmission. 
We require that if $A, B$ both follow the protocol, then both players accept except with probability negligible in the security parameter $k$. Also, whenever $A$ is honest, we have that $b_{A}$ is uniformly random and $b_{A}=s_{A}$. Whenever $B$ is honest, we have $r_{B}=b_{B}$. When $A$ is corrupted and $B$ is honest, we let $p_{A}$ be the probability of the event that $B$ accepts and $b_{A} \neq s_{A}$. Similarly, when $B$ is corrupted and $A$ is honest, we let $p_{B}$ be the probability that $A$ accepts and $r_{B} \neq b_{B}$. In general, the error probabilities $p_{A}, p_{B}$ will be functions of $\gamma, \delta$ and the security parameter $k$.

The argument sketched in [7] on constructing OT from UNC took as point of departure a protocol that builds OT from a $(\gamma, \delta)$-PassiveUNC for certain values of $\gamma, \delta$ and is secure assuming that players cheat only passively, i.e., are honest, but curious. It was then noted that one can replace the PassiveUNC with a UNC, still assuming that only passive cheating occurs. The final idea was then to replace the UNC with a committed UNC (although this notion was not formally defined there) and have players prove in ZK that they were following the protocol. If the error probabilities of the committed UNC could be made arbitrarily small with increasing $k$, then this would result in an OT secure against active cheating for essentially the same values of $\gamma, \delta$ that could be handled in the passive case. But unfortunately, this is impossible:

Theorem 1. Any committed UNC as defined above, based on a $(\gamma, \delta)$-UNC must have $p_{A}, p_{B} \geq \frac{\delta-\gamma}{1-2 \gamma}$.

Proof. Suppose, for instance, that $A$ is cheating. Then $A$ sets always the minimal noise level for the UNC, but adds artificial noise to each transmission with noise rate $\frac{\delta-\gamma}{1-2 \gamma}$ such that the total error probability for each transmission is $\frac{\delta-\gamma}{1-2 \gamma} \boxplus \gamma=\delta$. On the resulting transmissions, he runs a copy $A_{0}$ of the honest algorithm for $A$. Clearly, $B$ (who is honest) cannot distinguish this from an all honest situation where the noise rate happens to be $\delta$ all the time, and so he must accept with overwhelming probability. However, it now holds for every transmission that the bit committed to and also sent by $A_{0}$, differs from the one $A$ actually sent with probability $\frac{\delta-\gamma}{1-2 \gamma}$. The theorem follows.

Theorem 1 essentially says that we cannot force a player to commit to the bit he physically sends on a UNC. To get around this problem, we take a different point of view: we will create a new virtual channel from the UNC, where a bit committed to by the sender is by definition the bit sent on the new channel. Any difference between the committed bit and what is sent on the original UNC is regarded as noise. With appropriate checking that a cheating player does not introduce too much noise this way, it turns out that we obtain something that behaves as essentially like a PassiveUNC, even in presence of active cheating. We model this by an ideal functionality called $(\gamma, \delta, q())$-Committed PassiveUNC (CPUNC). It combines a functionality similar to the PassiveUNC with the Commit-and-Prove functionality. In particular, it allows to commit to bits with or without sending them on the channel. But if they are sent, sender and receiver will be committed to what they send/receive. With security parameter $k$, the error rate will be in the range $\delta \pm 1 / q(k)$, but will drop to $\gamma$ 
given the view of a cheating player. Note that a CPUNC is not a committed UNC, and so Theorem 1 does not forbid the existence of a secure implementation.

\section{Functionality $(\gamma, \delta, q())$-CPUNC}

Stop. On receiving this command from the adversary, the CPUNC stops working and ignores all further commands.

Send $c I D, b$ : CPUNC comes with parameters $0 \leq \gamma \leq \delta \leq 1 / 2$, a security parameter value $k$ and a polynomial $q()$. The issuer of the Send command is called the sender, the other party is the receiver. The string $c I D$ must not have been used before to identify a sent, received or committed bit, else the command is ignored. On receipt of this command from $A$ or $B$, the functionality records $c I D, b$ and outputs a string "which error probability?" to the adversary, it ignores all further commands until the adversary sends an "Error probability" command.

Error probability $\kappa^{\prime}$ : Receiving this command from the adversary, the functionality checks if $\left|\delta-\kappa^{\prime}\right| \leq 1 / q(k)$. If not, the command is ignored. Otherwise, the functionality chooses random bits $b^{\prime}, b^{\prime \prime}$, such that $\operatorname{Pr}\left(b^{\prime}=1\right)=\gamma$ and $\operatorname{Pr}\left(b^{\prime \prime}=1\right)=\nu$, where $\nu \boxplus \gamma=\kappa^{\prime}$. This ensures that $\operatorname{Pr}\left(b^{\prime} \oplus b^{\prime \prime}=1\right)=\kappa^{\prime}$. The functionality sets $\hat{b}=b \oplus b^{\prime} \oplus b^{\prime \prime}$. If the adversary has corrupted a player, it sends to the adversary a bit $z$, where $z=b \oplus b^{\prime \prime}$ if the sender is corrupted, and $z=b \oplus b^{\prime}$ if the receiver is corrupted. It records $c I D, b$ as if the sender had committed to $b$. It then sends $c I D$ to all players, and ignores all further commands until the receiver sends a "ReciptID" command.

ReceiptID $c \hat{I D}$ : This command is ignored if $c \hat{I D}$ has been used to identify any committed, sent or received bit earlier. If this is not the case, the CPUNC records $c \hat{I} D, \hat{b}$ as if the receiver had committed to $\hat{b}$, it sends $c \hat{I} D$ to all players and $\hat{b}$ to the receiver.

Commit $c I D, b$ : Receiving this command, where $c I D$ is a bitstring and $b$ is a bit, do as follows: if $c I D$ has not been used to identify a sent, received or committed bit before, record the value of $c I D, b$ and send as output Commit, $c I D$ to all players.

Open $c I D$ : if $c I D, b$ has been recorded as a commitment from the player issuing this command, send $b$ to all players.

Prove $L, \Phi$ : Receiving this command, where $L$ is a list of bit strings and $\Phi$ is a Boolean formula, check if $L$ contains only strings that has been used as identifiers for bits committed to by the issuer of the Prove command. If so, find the corresponding bits and check if they satisfy $\Phi$. If so, sends $(O K, L, \Phi)$ to all players. Else, send (Fail, $L, \Phi)$.

We now describe a protocol that securely realizes the functionality we just described. We assume that the protocol has access to the UNC, CaP and RandomChoice functionalities. The protocol is described by specifying how each of the commands are implemented. The amount of work done in the protocol is specified by a polynomial $p(k)$, where $k$ is the security parameter. 
Stop. This command has no direct implementation, the idea is that whenever the adversary behaves such that the honest party detects cheating and aborts, this is equivalent to sending a Stop command in the ideal scenario.

Send (Transmission Step). We describe how $A$ will send a bit $b$ to $B$.

1. $A$ commits to $b$

2. $A$ chooses at random bits $\bar{B}=b_{1}, \ldots, b_{k p(k)^{3}}$, commits to each bit and sends each bit to $B$ over the UNC. $B$ commits to every bit $\hat{B}=\hat{b}_{1}, \ldots, \hat{b}_{k p(k)^{3}}$ he receives.

3. Call RandomChoice $k p(k)^{2}$ times to generate integers $j_{i}$ chosen uniformly in the range $\left[1 . . k p(k)^{3}\right]$, for $i=1, \ldots, k p(k)^{2}$.

4. All bits $b_{j_{i}}, \hat{b}_{j_{i}}$ are opened. Let $\kappa$ be the fraction of the $k p(k)^{2}$ opened positions where $b_{j_{i}} \neq \hat{b}_{j_{i}}$. A and $B$ check that $\kappa \leq \delta+1 / p(k)$. They abort all interaction if this is not satisfied.

5. Call RandomChoice to generate an integer $j$ uniformly chosen among the indices of positions that were not opened in the previous step. $A$ sends $b^{\prime}=b \oplus b_{j}$ using error free transmission and proves (using CaP) that this value is correct.

6. Let $\mu$ be defined by $\kappa \boxplus \mu=\delta+1 / p(k)$. By a call to RandomChoice, generate a bit $c$ such that $\operatorname{Pr}(c=1)=\mu$.

7. $B$ defines the bit he receives as $\hat{b}=\hat{b_{j}} \oplus b^{\prime} \oplus c$. He commits to $\hat{b}$ and proves (using $\mathrm{CaP}$ ) that the committed value is correct.

If $B$ wants to send a bit to $A$, we implement this in the same way as above, by interchanging the roles of $A$ and $B$ and of $b_{j}$ and $\hat{b}_{j}$.

Commit, Open, Prove. Each of these commands correspond directly to commands that are already available in the Commit-and-Prove functionality we assume we have access to. Therefore these commands are implemented by directly calling the corresponding command with the same input in the Commit-and-Prove. Note that inputs to the Prove or Open command may include bits that were sent or received during a Send command, since these are also committed to.

Before proving anything about this construction, we describe first the intuition behind it: for bit strings $X, Y$ of equal length, let $\operatorname{err}(X, Y)$ be the fraction of positions where $X$ disagrees with $Y$. Now, if both parties are honest, the expected value of $\operatorname{err}(\bar{B}, \hat{B})$ is at most $\delta$, so allowing the estimate $\kappa$ to be up to $\delta+1 / p(k)$ implies that we reject with negligible probability, as we shall see. Then assume that one player, say $A$, is corrupted, and let $\tilde{B}=\tilde{b}_{1}, \ldots, \tilde{b}_{k p(k)^{3}}$ be the bits actually sent by $A$ on the UNC when a bit is transmitted. Let $\epsilon=\operatorname{err}(\bar{B}, \tilde{B})$. Since the UNC introduces errors with probability $\gamma$ independently of anything else, we expect that $\epsilon \boxplus \gamma \approx \operatorname{err}(\bar{B}, \hat{B}) \approx \kappa$, and hence that $\epsilon \boxplus \gamma \boxplus \mu \approx \kappa \boxplus \mu \approx \delta$. Here, $\approx$ means equality up to a $1 / \operatorname{poly}()$ term.

We can now see that after doing the transmission step, $A$ is actually in a position approximately equivalent to having sent $b$ on a $(\gamma, \delta)$-PassiveUNC: we have that the bit $b$ sent is related to the bit $\hat{b}$ received as $b=\hat{b} \oplus\left(b_{j} \oplus \tilde{b}_{j}\right) \oplus c \oplus n_{j}$, where $n_{j}$ is a noise bit chosen by the UNC, such that $\operatorname{Pr}\left(n_{j}=1\right)=\gamma$. By the 
choice of $c$, and random choice of $j$, we have

$$
\operatorname{Pr}(b \neq \hat{b})=\operatorname{Pr}\left(\left(b_{j} \oplus \tilde{b}_{j}\right) \oplus c \oplus n_{j}=1\right) \approx \epsilon \boxplus \mu \boxplus \gamma \approx \kappa \boxplus \mu \approx \delta .
$$

But since the adversary knows $\left(b_{j} \oplus \tilde{b}_{j}\right) \oplus c$, the error rate from his point of view is only what is introduced by the UNC, namely $\gamma$.

In the full paper [6] we show the theorem below. First, some terminology to state the result: we say that a simulator (in the UC framework) is non-blocking, if it stops the CPUNC (by sending a stop command or refusing to give correct input when asked for it) with only negligible probability.

Theorem 2. The Committed Passive UNC protocol securely realizes the $(\gamma, \delta, q())-C P U N C$ functionality when given access to ideal $(\gamma, \delta)-U N C, C a P$ and RandomChoice functionalities, and for any polynomial $q()$, provided we choose the polynomial $p()$ measuring the work done in the protocol as $p(k)=4 q(k)$. Moreover, for the case where both players are honest, the simulator is nonblocking.

Remark 1. The last claim in the theorem is a way to state in the UC framework the traditional completeness property for a 2-party protocol: if both players are honest, the protocol completes successfully with overwhelming probability.

\section{From Passive to Active Security}

In this section, we sketch a proof of the following result:

Theorem 3. Let $\pi$ be any protocol that securely realizes OT based on a $(\gamma, \delta)$ PassiveUNC assuming a passive adversary. Then there exists a protocol with complexity polynomial in that of $\pi$ that also securely realizes OT based on a $(\gamma, \delta)-U N C$, assuming an active adversary.

So we assume we have a protocol $\pi$ that implements Oblivious Transfer given access to a $(\gamma, \delta)$-PassiveUNC functionality, and that this protocol is secure against a passive adversary.

We then note that the previous section showed how to implement the CPUNC functionality based on the UNC. Therefore from $\pi$, we may construct a protocol $\bar{\pi}$ as follows: active cheating is prevented by first making players commit to all inputs, and furthermore, the random coins of a player are decided using a standard trick: the player in question commits to a random string $a$, the other player sends a random string $b$ in the clear and the random coins to be used are $a \oplus b$. Second, all transmissions over the PassiveUNC now take place using the CPUNC, and each time something is sent, you use the CPUNC to prove that what was sent was computed according to $\pi$ with the given (committed) inputs, random coins and messages received earlier.

Note that a player trying to send an incorrect message will be caught with certainty. Therefore, the views obtained by the players are always (a possibly truncated version of) what would be obtained in presence of a passive adversary.

Our first goal will be to show that $\bar{\pi}$ implements a weak form of OT (which then implies standard OT), namely a $(p, q, \epsilon)$-WOT as defined in Section 3 
Lemma 1. $\bar{\pi}$ as described above realizes (with statistically good simulation) a $(p, q, \epsilon)$-WOT with $p=q=\epsilon=3 / k$, when $\bar{\pi}$ is executed with security parameter value $k$.

Proof. (Sketch) The above discussion implies that we only have to show the lemma for a passive adversary: the only difference between a passive and an active attack on $\bar{\pi}$ is that the adversary may stop early in the active case, and this can never be prevented in an active attack. Assuming a passive adversary, the only difference between $\bar{\pi}$ and $\pi$ is that $\bar{\pi}$ does not use a $(\gamma, \delta)$-PassiveUNC but a $(\gamma, \delta, f())$-CPUNC where the adversary can make the error probability fluctuate slightly around $\delta$. This fluctuation is not negligible, namely it is of size $1 / f(k)$. However, by Theorem [2, we can choose $f()$ to be any polynomial we like, so assuming $\pi$ calls the PassiveUNC $t(k)$ times, for some polynomial $t()$, we choose $f(k)=k t(k)$.

Consider the view of a (passively) corrupted sender in $\pi$, represented by random variable $V$. Let $a d v_{\pi}(k, v)$ be the advantage over $1 / 2$ with which the selection bit can be guessed given that $V=v$ and the protocol was executed with security parameter value $k$. Let $a d v_{\pi}(k)=\sum_{v} \operatorname{Pr}(V=v) \cdot a d v_{\pi}(k, v)$ be the expected value. Since $\pi$ was assumed to be secure, $a d v_{\pi}(k)$ is negligible in $k$ (this is equivalent to asserting that the mutual information between the selection bit and $V$ is negligible). Then define a particular possible value $v$ of $V$ to be $\operatorname{good}$ if $a d v_{\pi}(k, v) \leq \sqrt{a d v_{\pi}(k)}$, and let $E$ be the event that $V$ takes a bad value. Then clearly, $E$ occurs with probability at most $\sqrt{a d v_{\pi}(k)}$. We now define $t(k)+1$ hybrids that are in between $\pi$ and $\bar{\pi}$ : namely in the $i$ 'th hybrid, where $i=0 . . t(k)$, we run the normal protocol, but for communication, we use a $(\gamma, \delta)$-PassiveUNC for the first $i$ calls to the communication channel, and then the $(\gamma, \delta)$-CPUNC for the rest. Then hybrid 0 is $\bar{\pi}$ while hybrid $t(k)$ is $\pi$. When executing hybrid $i$, we define $E_{i}$ to be the event that the information contained in the sender's view about the selection bit is larger than $\sqrt{a d v_{\pi}(k)}$. Let $\epsilon_{i}$ be the probability that $E_{i}$ occurs. Of course $\epsilon_{t(k)}=\operatorname{Pr}(E) \leq \sqrt{a d v_{\pi}(k)}$. Also, the only difference between hybrid $i$ and $i+1$ is that in the $i+1$ 'st call to the communication channel, the results returned by the channel have distributions with statistical difference at most $2 / f(k)$ between them. It follows that $\left|\epsilon_{i}-\epsilon_{i+1}\right| \leq 2 / f(k)$, and hence $\epsilon_{0} \leq \epsilon_{t(k)}+2 t(k) / f(k) \leq \sqrt{a d v_{\pi}(k)}+2 / k$. The "OT", that $\bar{\pi}$ implements is therefore no worse than a protocol that with probability, say $3 / k$ reveals the selection bit to the sender, and otherwise leaks a negligible amount of information. A similar argument holds for the view of a corrupted receiver; also this type of argument shows that an honest receiver will receive the correct bit, except with probability at most $3 / k$. Thus what we have is statistically indistinguishable from a $(p, q, \epsilon)$-WOT, with $p=q=\epsilon=3 / k$.

We can then complete the argument for the theorem: In [7, a reduction is shown that implements OT based on any $(p, q, \epsilon)$-WOT, as long as $p+q+2 \epsilon<$ 0.45 . Moreover, it is easy to verify that by choosing $k$ large enough the reduction implements OT efficiently, i.e., it only makes a polynomial number of calls to the underlying WOT. Therefore, by the above lemma, we can replace the WOT 
by $\bar{\pi}$ and still obtain a secure OT (even though $\bar{\pi}$ is only statistically close to the required WOT). This implies the result we wanted.

\section{Extended Positive Results}

In this section, we shall assume the result of Theorem 3 and focus on reducing OT to $(\gamma, \delta)$-PassiveUNC securely against passive adversaries. The strategy of [7] is as follows. First, the $(\gamma, \delta)$-PassiveUNC is used to construct an imperfect version of OT which may leak information about the parties' private inputs. This imperfect OT is modeled by a WOT. OT is then shown to be reducible to WOT for certain values of $(\gamma, \delta)$.

However, WOT does not precisely capture the imperfect OT obtained in the construction: In WOT the corrupted sender/receiver gets the selection/secret bit (which he is not supposed to see) with a certain probability, while in the imperfect OT obtained the corrupted sender/receiver only gets some information about that bit with a certain probability. As a consequence, in order to fit the imperfect OT into the WOT model, it is assumed in [7] that every time the dishonest sender/receiver gets some information about the selection/secret bit, he actually gets full information. Hence, the information leakage is overestimated in [7]. We introduce a new Generalized Weak Oblivious Transfer (GWOT) primitive which allows to model imperfect OTs which leak information about the parties' private inputs in a much more general way than WOTs, without overestimating the information leakage. In particular, it precisely captures the imperfect OT resulting from the construction of [7]. Informally, in a GWOT the corrupted sender/receiver gets the selection/secret bit over a BSC with some error probability which is chosen according to some distribution (and announced to the corrupted party). Formally, consider parameters $\left\{s_{i}, \alpha_{i}\right\}_{i}$ and $\left\{r_{i}, \beta_{i}\right\}_{i}$, where $i=1, \ldots, N$, and $\epsilon$ such that $\left\{s_{i}\right\}_{i}$ and $\left\{r_{i}\right\}_{i}$ are probability distributions (over $\{1, \ldots, N\}$ ) and $0 \leq \alpha_{i}, \beta_{i}, \epsilon \leq 1 / 2$ for $i=1, \ldots, N$. A GWOT with respect to these parameters is specified by a functionality of the following kind. Functionality $\left(\left\{\left(s_{i}, \alpha_{i}\right)\right\}_{i=1}^{N} ;\left\{\left(r_{i}, \beta_{i}\right)\right\}_{i=1}^{N} ; \epsilon\right)$-GWOT

Send $\left(b_{0}, b_{1}\right)$ : The functionality's action on this command is the same as in OT. Choice $c$ : If $c \notin\{0,1\}$ then the functionality ignores the command. Otherwise, it chooses $\tilde{b}_{c} \in\{0,1\}$ such that $\operatorname{Pr}\left(\tilde{b}_{c} \neq b_{c}\right)=\epsilon$ and sends it to the receiver. Additionally, if the sender is corrupted, then it chooses $I \in\{1, \ldots, N\}$ and $\tilde{c} \in\{0,1\}$ such that $\operatorname{Pr}(I=i)=s_{i}$ and $\operatorname{Pr}(\tilde{c} \neq c \mid I=i)=\alpha_{i}$, and it sends $I$ and $\tilde{c}$ to the sender. And/or, if the receiver is corrupted, then it chooses $I \in\{1, \ldots, N\}$, and $\tilde{b}_{1-c} \in\{0,1\}$ such that $\operatorname{Pr}(I=i)=r_{i}$ and $\operatorname{Pr}\left(\tilde{b}_{1-c} \neq b_{1-c} \mid I=i\right)=\beta_{i}$, and it sends $I$ and $\tilde{b}_{1-c}$ to the receiver.

We will say that a corrupted sender gets $c$ "sent through $\left\{\left(s_{i}, \alpha_{i}\right)\right\}_{i=1}^{N}$ " and similarly a corrupted receiver gets $b_{1-c}$ "sent through $\left\{\left(r_{i}, \beta_{i}\right)\right\}_{i=1}^{N}$ ".

Note that there is some ambiguity in the functionality's action in that it is not required that $\tilde{b}_{c}$ is chosen independently of $I$ and $\tilde{c}$, respectively of $I$ and $\tilde{b}_{1-c}$, as 
long as the marginal distribution of $\tilde{b}_{c}$ is correct. Furthermore, a $(p, q, \epsilon)$-WOT coincides obviously with a $(\{(p, 0),(1-p, 1 / 2)\} ;\{(q, 0),(1-q, 1 / 2)\} ; \epsilon)$-GWOT.

It will be convenient to introduce a GWOT of a very particular form, a Special Generalized Oblivious Transfer (SGWOT). Informally, in a SGWOT the corrupted sender/receiver either gets no information on the selection/secret bit or he receives it over a BSC with a certain (fixed) error probability. Formally, for parameters $s, \alpha, r, \beta, \epsilon$ with $0 \leq s, r \leq 1$ and $0 \leq \alpha, \beta \leq 1 / 2$,

$$
((s, \alpha),(r, \beta), \epsilon) \text {-SGWOT } \stackrel{\text { def }}{=}(\{(s, 1 / 2),(1-s, \alpha)\} ;\{(r, 1 / 2),(1-r, \beta)\} ; \epsilon) \text {-GWOT. }
$$

Consider the reduction of WOT to $(\gamma, \delta)$-PassiveUNC given in Appendix A of [7]. As mentioned above, this construction actually results in a GWOT (which is modeled by a WOT by giving away information to the adversary). As a matter of fact, as can easily be seen, it results in a SGWOT. The following Lemma expresses the parameters of the resulting SGWOT as a function of $(\gamma, \delta)$. For convenience, we write $\mu=\frac{\delta-\gamma}{1-2 \gamma}$, such that $\gamma \boxplus \mu=\delta$. The proof of the Lemma follows by straightforward analysis of reduction WOTfromPassiveUNC of [7].

Lemma 2. When run with a $(\gamma, \delta)$-UNC, reduction WOTfromPassiveUNC defined in 7 produces a $((s, \alpha),(r, \beta), \epsilon)-S G W O T$ with the following parameters:

$$
\begin{gathered}
s=\frac{\gamma(1-\gamma)\left(\gamma^{2}+(1-\gamma)^{2}\right)\left(\mu^{4}+6 \mu^{2}(1-\mu)^{2}+(1-\mu)^{4}\right)}{\delta(1-\delta)\left(\delta^{2}+(1-\delta)^{2}\right)}, \alpha=\frac{4 \gamma^{2}(1-\gamma)^{2}}{\gamma^{4}+6 \gamma^{2}\left(1-\gamma^{2}\right)+\left(1-\gamma^{4}\right)} \\
r=\frac{\gamma(1-\gamma)\left(\mu^{2}+(1-\mu)^{2}\right)}{\delta(1-\delta)}, \beta=\frac{\gamma^{2}}{\gamma^{2}+(1-\gamma)^{2}} \\
\epsilon=\frac{\delta^{2}}{\delta^{2}+(1-\delta)^{2}} .
\end{gathered}
$$

We have expressed the parameters of $((s, \alpha),(r, \beta), \epsilon)$-SGWOT with that of the underlying $(\gamma, \delta)$-PassiveUNC. Now we would like to exploit the machinery of [7] in order to reduce OT to SGWOT. A composition of three basic reductions is used in order to transform a WOT into an OT. The first reduction, S-Red $(l)$ decreases the sender's information about the selection bit by executing WOT $l$ times such that the final selection bit is the parity of all selection bits used during the $l$ executions (this reduction was introduced in [5]). The second reduction, R-Red $(l)$, decreases the receiver's information about the bit that was not selected by encoding it into the parity of $l$ transmissions. The final reduction, E-Red $(l)$, decreases the error rate by executing $l$ identical transmissions through a WOT. Every of these reductions transforms the WOT into a new one (with new parameters), and it is shown in [7] that for certain initial parameters the sequence of WOTs converges to an OT (in some well defined meaningful sense).

In [7], the $((s, \alpha),(r, \beta), \epsilon)$-SGWOT obtained after invoking WOTfromPassiveUNC was modeled by a $(1-s, 1-r, \epsilon)$-WOT. I.e., in order to fit the imperfect OT into the WOT framework, the error probabilities $\alpha$ and $\beta$ were assumed to be zero by giving the corrupted party some information for free. Clearly, a tighter analysis should avoid this kind of strengthening of the corrupted party for proof-technical conveniences. A straight forward approach would be to try to 
show that for certain initial parameters, the sequence of GWOTs, resulting by applying the S-, R- and E-Red reductions to the initial SGWOT, converges to an OT. Unfortunately, as the reduction of OT to WOT defined in [7] is executed, the shape of the GWOTs becomes quickly very complex and difficult to analyze. In order to avoid this problem, we give a generic way to replace a (possibly very complex) GWOT by another (ideally simpler) one such that if the new GWOT allows for OT then the initial GWOT also allows for OT; however, in contrast to the strategy of [7] of simply setting the error probabilities to zero, we are trying to be much more tight.

Next definition introduces a partial ordering "§” among probability distributions over BSCs, i.e. among sets of the form $\left\{\left(s_{i}, \alpha_{i}\right)\right\}_{i}$ or $\left\{\left(r_{i}, \beta_{i}\right)\right\}_{i}$ as considered above, that will be shown (in Lemma 3) to capture the relative difficulty to generate OT using the reduction considered in [7. Intuitively, we say that $S \preceq S^{\prime}$ if $S$ can be transformed into $S^{\prime}$ by removing BSCs in $S$ and replacing each of them by a Bernoulli distribution over 2 BSCs such that the average guessing probability for the bit sent through $S$ is the same as when sent through $S^{\prime}$.

Definition 1. Let $S=\left\{\left(p_{i}, \epsilon_{i}\right)\right\}_{i=1}^{N}$ and $S^{\prime}$ be two probability distributions over $B S C$. We say that $S \preceq S^{\prime}$ if there exists $1 \leq \ell \leq N$ as well as $0 \leq \delta \leq 1$ and $0 \leq \epsilon^{-} \leq \epsilon \leq \epsilon^{+} \leq 1 / 2$ such that

1. $S^{\prime}$ is of the form $S^{\prime}=S \backslash\left\{\left(p_{\ell}, \epsilon_{\ell}\right)\right\} \cup\left\{\left((1-\delta) p_{\ell}, \epsilon^{-}\right),\left(\delta p_{\ell}, \epsilon^{+}\right)\right\}$and 2. $\epsilon_{\ell}=\epsilon=(1-\delta) \cdot \epsilon^{-}+\delta \cdot \epsilon^{+}$,

or if there exists a sequence $S=S_{0}, S_{1}, \ldots, S_{k}=S^{\prime}$ of probability distributions over BSCs such that $S_{\kappa-1} \preceq S_{\kappa}$ in the above sense for $\kappa=1, \ldots, k$.

Note that in case $\epsilon_{j}=\epsilon_{k}$ for some $1 \leq j<k \leq N$, we identify $S=\left\{\left(p_{i}, \epsilon_{i}\right)\right\}_{i=1}^{N}$ with $S^{*}=S \backslash\left\{\left(p_{j}, \epsilon_{j}\right),\left(p_{k}, \epsilon_{k}\right)\right\} \cup\left\{\left(p_{j}+p_{k}, \epsilon_{j}\right)\right\}$. This is justified in that it is immaterial in our context whether a bit is sent thorough $S$ or through $S^{*}$.

The next lemma, a proof of which can be found in the full paper [6], shows that the partial ordering $S \preceq S^{\prime}$ means that as long as reductions S-Red, R-Red, and E-Red are concerned, $S$ is easier to deal with than $S^{\prime}$.

Lemma 3. If OT can be reduced to $\left(S^{\prime} ; R^{\prime} ; \epsilon\right)$-GWOT by a sequence of reductions $S$-Red, R-Red, and E-Red, then $O T$ can be reduced to any $(S ; R ; \epsilon)-G W O T$ with $S \preceq S^{\prime}$ and $R \preceq R^{\prime}$.

One application of Lemma 3 allows to improve the analysis of [7]. As we have seen in Lemma 2. the imperfect OT obtained from a UNC using reduction WOTfromPassiveUNC produces a $((s, \alpha),(r, \beta), \epsilon))$-SGWOT. Using Lemma 3 it is straightforward to verify that we can replace this SGWOT by a $\left(p_{s}, q_{r}, \epsilon\right)$ WOT with $p_{s}=(1-s)(1-2 \alpha)$ and $q_{r}=(1-r)(1-2 \beta)$. Indeed, for instance the corrupted sender's guessing probability for the selection bit is in the first case $s / 2+(1-s)(1-\alpha)=1-s / 2-\alpha+s \alpha$ and in the second case $p_{s}+\left(1-p_{s}\right) / 2=1-s / 2-\alpha+s \alpha$. Applying Lemma 5 of [7] (OT is possible based on $(p, q, \epsilon)$-WOT if $p+q+2 \epsilon \leq 0.45)$ to the transformed SGWOT results in the following Lemma. 
Lemma 4. The reduction from OT to WOT of [7] implements OT from any $((s, \alpha),(r, \beta), \epsilon)-S G W O T$ with $p_{s}+q_{r}+2 \epsilon \leq 0.45$, where $p_{s}=(1-s)(1-2 \alpha)$ and $q_{r}=(1-r)(1-2 \beta)$.

Combining Lemmas 4 and 2 gives directly the following result:

Lemma 5. OT may be reduced to $(\gamma, \delta)$-PassiveUNC if $p_{s}+q_{r}+2 \epsilon \leq 0.45$, where $p_{s}=(1-s)(1-2 \alpha), q_{r}=(1-r)(1-2 \beta)$ and $s, \alpha, r, \beta, \epsilon$ are defined by equations (1)- (3).

Note that [7] only guarantees that OT can be achieved if $p+q+2 \epsilon \leq 0.45$ where $p=1-s$ and $q=1-r$. Hence, the possibility range given in Lemma 5 strictly contains the one obtained in [7.

Despite this improvement, Lemma 5 still shares the following restriction with 77. OT cannot be provably achieved for $\delta>0.35$ even when $\gamma$ is almost equal to $\delta$ (i.e. the resulting UNC has almost no unfairness) since in that case $\epsilon>0.45$ (see Figure 1). This stands somewhat in contrast to the fact that OT can be achieved based on any (non-trivial) BSC 412[15]. Hence, one would expect that OT can be achieved based on any (non-trivial) UNC as long as the unfairness is small enough. The following lemma shows that this is indeed true.

Lemma 6. There exists a reduction from OT to any $(\gamma, \delta)$-PassiveUNC that satisfies $1-\left(1-p_{s}\right)^{l}+1-\left(1-q_{r}\right)^{l}+2 \frac{\epsilon^{l}}{\epsilon^{l}+(1-\epsilon)^{l}} \leq 0.45$ for some $l \geq 1$, where $p_{s}=(1-s)(1-2 \alpha)$ and $q_{r}=(1-r)(1-2 \beta)$ with $s, \alpha, r, \beta, \epsilon$ defined by (1)-(3).

Clearly, for any $0<\delta<1 / 2$, for $l$ large enough, and for $\gamma$ close enough to $\delta$ (where the closer $\delta$ is to $1 / 2$, the closer $\gamma$ has to be to $\delta$ ), the values $p_{s}$ and $q_{r}$ are small enough for the condition expressed in Lemma 6 to be satisfied. Hence, OT is possible based on $(\gamma, \delta)$-PassiveUNC's for any $0<\delta<1 / 2$ as long as $\gamma$ is close enough to $\delta$ (see Figure 1). This further improves on [7].

Proof. We implement a $((s, \alpha),(r, \beta), \epsilon)$-SGWOT from the $(\gamma, \delta)$-PassiveUNC according to Lemma 2 Then, by Lemma 3, we convert it into a $\left(p_{s}, q_{r}, \epsilon\right)$-WOT before applying the reduction $\operatorname{E-Red}(l)$ [7] with parameter $l$. As shown in [7], this results in a $\left(1-\left(1-p_{s}\right)^{l}, 1-\left(1-q_{r}\right)^{l}, \frac{\epsilon^{l}}{\epsilon^{l}+(1-\epsilon)^{l}}\right)$-WOT. The claim now follows from the above.

It can be shown by straightforward calculations that the new possibility range includes UNC's for which the techniques of [7] results in a "simulatable" WOT (i.e., a trivial WOT), that is, could not be used to implement OT (see Lemma 1 from [7]). In other words, our approach allows to implement and prove secure OT in a range where it is provably impossible using the techniques of [7]. The following example illustrates this.

Example 1. Let $\gamma_{0}=0.39, \delta_{0}=0.4$ be the parameters of a PassiveUNC. The $\left(p\left(\gamma_{0}, \delta_{0}\right), q\left(\gamma_{0}, \delta_{0}\right), \epsilon\left(\delta_{0}\right)\right)$-WOT obtained from a $\left(\gamma_{0}, \delta_{0}\right)$-PassiveUNC the crude way (by giving away all partial information to the adversary as in 7]) achieves $p\left(\gamma_{0}, \delta_{0}\right)+q\left(\gamma_{0}, \delta_{0}\right)+2 \epsilon\left(\delta_{0}\right) \approx 0.869$. It can be shown that from this WOT, any 
sequence of reductions S-, R- and E-Red generates a simulatable WOT, i.e., OT is not reducible to the $\left(p\left(\gamma_{0}, \delta_{0}\right), q\left(\gamma_{0}, \delta_{0}\right), \epsilon\left(\delta_{0}\right)\right)$-WOT using S-, R- and E-Red. At the same time, the $\left(p_{s}\left(\gamma_{0}, \delta_{0}\right), q_{r}\left(\gamma_{0}, \delta_{0}\right), \epsilon\left(\delta_{0}\right)\right)$-WOT (obtained according Lemma 3) achieves $p_{s}\left(\gamma_{0}, \delta_{0}\right)+q_{r}\left(\gamma_{0}, \delta_{0}\right)+2 \epsilon\left(\delta_{0}\right) \approx 0.671$. Moreover, E-Red $(2)$ applied to this WOT generates a $\left(p^{\prime}, q^{\prime}, \epsilon^{\prime}\right)$-WOT with $p^{\prime}+q^{\prime}+2 \epsilon^{\prime} \approx 0.438$, which we know from Lemma 5 implies OT.

There exists an even larger range than the one described in Lemma 6 for which a possibility result can be shown. This follows from the fact that the approach of Lemma 6 still gives information for free to the adversary. Indeed, the SGWOT obtained from a $(\gamma, \delta)$-PassiveUNC is converted into a $\left(p_{s}, q_{r}, \epsilon\right)$ WOT before reductions S-Red, R-Red and E-Red are applied. We may benefit from trying preserving the SGWOT through the sequence of reductions.

The problem is that the reductions do not preserve the SGWOT per se but produce more complex GWOTs with a quickly growing set of parameters. An approach is to use Lemma 3 in order to immediately convert any resulting GWOT (which is not a SGWOT) back into a SGWOT. Specifically, a $\left(\left\{\left(s_{i}, \alpha_{i}\right)\right\}_{i} ;\left\{\left(r_{i}, \beta_{i}\right)\right\}_{i} ; \epsilon\right)$-GWOT can be replaced by a $((s, \alpha),(r, \beta), \epsilon)$-SGWOT, where $\alpha=\min _{i}\left\{\alpha_{i}\right\}$ and $\beta=\min _{i}\left\{\beta_{i}\right\}$, and $s$ and $r$ are appropriately chosen such that $\left\{\left(s_{i}, \alpha_{i}\right)\right\}_{i} \preceq\{(s, 1 / 2),(1-s, \alpha)\}$ and $\left\{\left(r_{i}, \beta_{i}\right)\right\}_{i} \preceq\{(r, 1 / 2),(1-r, \beta)\}$. This indeed results in an increased possibility range:

Lemma 7. There exists a range of values $(\gamma, \delta)$ which do not satisfy the conditions of Lemma 6 but where OT can still be implemented from such $(\gamma, \delta)-U N C$ 's.

Proof. (sketch) By brute force analysis for any fixed value of $\delta_{0}, 0<\delta_{0}<1 / 2$, we find the smallest value of $\gamma_{0}$, such that a SGWOT based on $\left(\gamma_{0}, \delta_{0}\right)$-PassiveUNC can be reduced to a SGWOT with $p_{s}+q_{r}+2 \epsilon \leq 0.45$ using the reductions S-Red, R-Red and E-Red, and replacing any GWOT by a SGWOT as sketched above.

For example, let $\gamma_{0}=0.365, \delta_{0}=0.4$. The value $p_{s}+q_{r}+2 \epsilon$ of the SGWOT resulting from $\left(\gamma_{0}, \delta_{0}\right)$-PassiveUNC is equal to 0.793 . It is easy to check that the conditions of Lemma 6 are not satisfied with respect to this SGWOT. Nonetheless, the sequence of reductions "EERSRESERRSESRERSESERRS" (each with parameter $l=2$ ) produces as output a SGWOT with $p_{s}+q_{r}+2 \epsilon=0.329$ which implies OT according Lemma 5 .

Using brute-force analysis, it is possible to find experimentally the range for which the reduction considered in Lemma 7 produces OT. The new range is depicted on Figure 1 .

On the other hand, even the approach described above is limited in power. The following example suggests that in order to get a possibility result closer to the $(\gamma, \delta)$-PassiveUNC simulation bound $\delta=2 \gamma(1-\gamma)$ from [7], one has to find different reduction methods and/or analytical tools.

Example 2. Let $\gamma_{0}=0.33, \delta_{0}=0.4$. A SGWOT based on $\left(\gamma_{0}, \delta_{0}\right)$-PassiveUNC has the potential $p_{s}\left(\gamma_{0}, \delta_{0}\right)+q_{r}\left(\gamma_{0}, \delta_{0}\right)+2 \epsilon\left(\delta_{0}\right) \approx 0.949$. It can be shown by brute force analysis that whatever sequence of reductions S-, R- and E-Reduce 


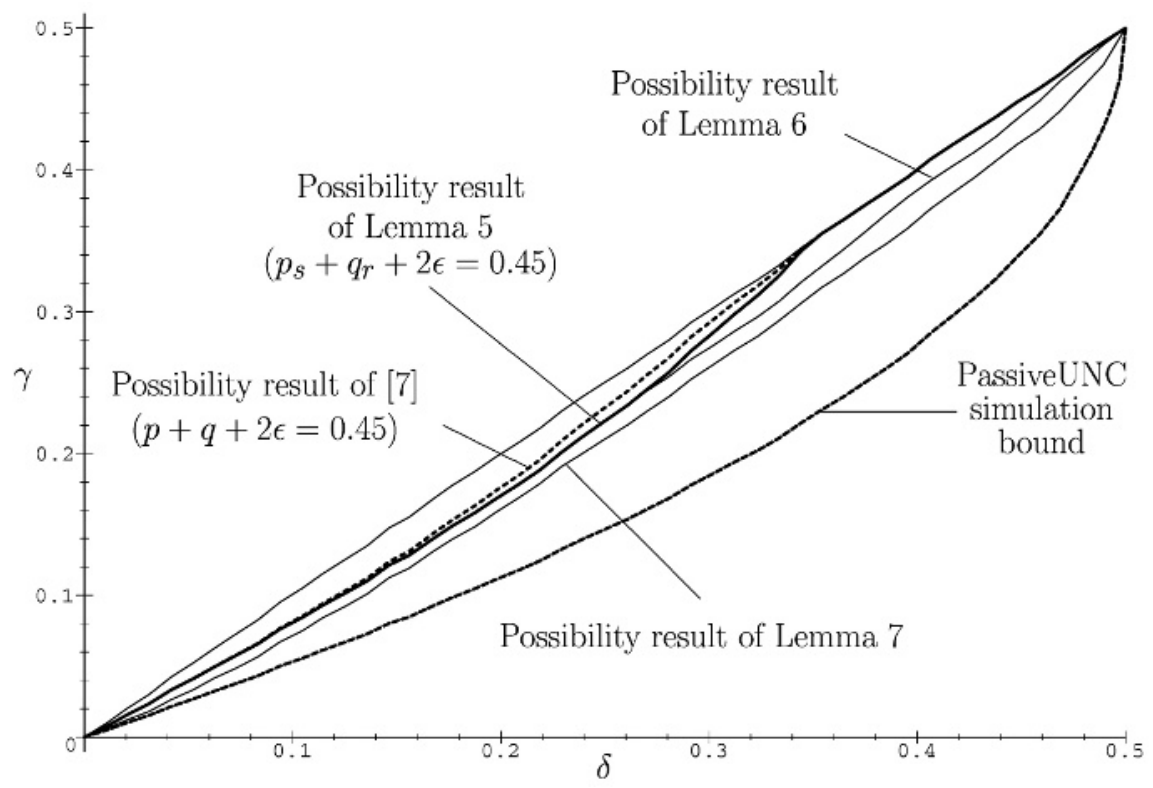

Fig. 1. Positive results on OT from $(\gamma, \delta)$-PassiveUNC

applied with whatever parameters, it always results at some point a SGWOT with $p_{s}+q_{r}+2 \epsilon \geq 1$.

We stress that in contrast to a $(p, q, \epsilon)$-WOT with $p+q+2 \epsilon \geq 1$, a SGWOT with $p_{s}+q_{r}+2 \epsilon \geq 1$ is not proven to be simulateable; however, it seems to be a very strong indication that OT cannot be based on such a SGWOT.

\section{Conclusion and Open Questions}

In this paper, we have shown how to transform any OT protocol secure against passive adversaries given access to a PassiveUNC into one that is secure against active adversaries given access to a standard UNC. This is possible since any non-trivial UNC allows for bit commitment as it was shown in 7]. Our transformation is general enough to be applicable to a wider class of 2-party protocols. Applying it to a passively secure protocol $\pi$ implementing task $T$ given access to a PassiveUNC produces an actively secure protocol $\pi^{\prime}$ that implements $T$ given access to a UNC, however, $\pi^{\prime}$ may fail with non-negligible (1/poly) probability. When $T$ is OT, this can be cleaned up using known techniques, in general $T$ can be any task where such "cleaning" is possible.

We have also provided a more refined analysis for the reduction of OT to $(\gamma, \delta)$-UNC introduced in [7]. As a result, OT is now possible based on a significantly larger range of $(\gamma, \delta)$ than what was known before. Unfortunately, we also show the approach has limits that even a more careful analysis cannot overcome. 
Thus, a grey area is left where no positive or negative results are known to apply. Closing this gap is the obvious open problem suggested by this work.

\section{References}

1. Brassard, G., Chaum, D., Crépeau, C.: Minimum Disclosure Proofs of Knowledge. J. of Computer and System Sciences, 37(2). Elsevier (1988) 156-189

2. Canetti, R.: Universally Composable Security: A New Paradigm for Cryptographic Protocols. In: 42nd Symposium FOCS, IEEE (2001) 136-145

3. Chaum, D., Damgård, I., van de Graaf, J.: Multi-party Computations Ensuring Privacy of Each Party's Input and Correctness of the Result. In: Advances in Cryptology-CRYPTO '87. LNCS, vol. 293. Springer-Verlag (1987) 462

4. Crépeau, C.: Efficient Cryptographic Protocols Based on Noisy Channels. In: Advances in Cryptology-EUROCRYPT'97. LNCS, vol. 1233. Springer-Verlag (1997) 306-317

5. Crépeau, C., Kilian, J.: Achieving Oblivious Transfer Using Weakened Security Assumptions. In: 29th Symposium FOCS, IEEE (1988) 42-52

6. Damgård, I., Fehr, S., Morozov, K. and Salvail, L.: Unfair Noisy Channels and Oblivious Transfer, full version of this paper, BRICS report nr. RS-03-36, (2003) available from www.brics.dk/RS/03/36

7. Damgård, I., Kilian, J., Salvail, L.: On the (Im)possibility of Basing Bit Commitment and Oblivious Transfer on Weakened Security Assumptions. In: Advances in Cryptology-EUROCRYPT '99. LNCS, vol. 1592. Springer-Verlag (1999) 56-73

8. Goldreich, O., Micali, S., Wigderson, A.: Proofs that Yield Nothing but the Validity of the Assertion, and the Methodology of Cryptographic Protocol Design. In: 27th Symposium FOCS. IEEE (1986) 174-187

9. Goldreich, O., Micali, S., Wigderson, A.: How to Play Any Mental Game. In: 19th ACM STOC. ACM Press (1987) 218-229

10. Kilian, J.: Founding Cryptography on Oblivious Transfer. In: 20th ACM STOC. ACM Press (1988) 20-31

11. Kilian, J.: A Note on Efficient Proofs and Arguments. In: 24th ACM STOC. ACM Press (1992) 723-732

12. Korjik, V., Morozov, K.: Generalized Oblivious Transfer Protocols Based on Noisy Channels. In: Proc. Workshop MMM ACNS 2001. LNCS, vol. 2052. SpringerVerlag (2001) 219-229

13. Lo, H.-K., Chau, H.F.: Is Quantum Bit Commitment Really Possible?. Physical Review Letters, vol. 78, no 17, (1997) 3410-3413

14. Mayers, D.: Unconditionally Secure Quantum Bit Commitment is Impossible. Physical Review Letters, vol. 78, no 17, (1997) 3414-3417

15. Stebila, D., Wolf, S.: Efficient Oblivious Transfer From Any Non-Trivial BinarySymmetric Channel. In: International Symposium on Information Theory (ISIT) (2002) 293 